\title{
Archipel
}

ARCHIPEL Études interdisciplinaires sur le monde insulindien

$89 \mid 2015$

Varia

\section{À la recherche de manuscrits malais en France, avec une mention toute particulière sur les lettres de Francis Light}

Jelani Harun

\section{(2) OpenEdition \\ Journals}

Édition électronique

URL : http://journals.openedition.org/archipel/488

DOI : 10.4000 /archipel.488

ISSN : 2104-3655

Éditeur

Association Archipel

\section{Édition imprimée}

Date de publication : 15 avril 2015

Pagination : 39-61

ISBN : 978-2-910513-72-6

ISSN : 0044-8613

Référence électronique

Jelani Harun, « À la recherche de manuscrits malais en France, avec une mention toute particulière sur les lettres de Francis Light », Archipel [En ligne], 89 | 2015, mis en ligne le, consulté le 05 mars 2021. URL : http://journals.openedition.org/archipel/488 ; DOI : https://doi.org/10.4000/archipel.488 
$J_{E L A N I} H_{A R U N}{ }^{1}$

\section{À la recherche de manuscrits malais en France, avec une mention toute particulière sur les lettres de Francis Light ${ }^{2}$}

Il existe en France une collection de manuscrits malais, venant numériquement en cinquième position après les Pays-Bas, l'Indonésie, la Grande-Bretagne et la Malaisie (Chambert-Loir, 1980a, p. 45) ${ }^{3}$. Ces manuscrits, sont, pour la plupart, conservés à Paris, principalement à la Bibliothèque nationale, mais aussi, en nombre beaucoup plus restreint, à Marseille, à Rouen et à Tournus. Lors d'une mission de recherche d'un mois en France, en juillet 2011, j'ai consulté les fonds de Paris et de Tournus. Dans cet article, je me propose de donner une vue générale de ces manuscrits. Je parlerai de la manière dont les collections ont été constituées, comment et par qui les manuscrits ont été répertoriés et où ils sont localisés. On verra que les premiers chercheurs et enseignants de l'École des Langues orientales vivantes ${ }^{4}$ ont participé activement à la constitution et au catalogage des manuscrits malais de la Bibliothèque nationale et qu'ils en utilisaient certains, les publiant, les étudiant, les traduisant, s'en servant pour leur enseignement.

1. School of Humanities, Universiti Sains Malaysia, Penang, Malaysia.

2. Cet article est adapté par Monique Zaini-Lajoubert d'une communication en malaisien de l'auteur intitulée « Menjejaki Manuskrip Melayu dan Surat-surat Francis Light di Perancis », Perpustakaan Negara Malaysia, 6 mai 2014. http://myrepositori.pnm.gov.my/xmlui/handle/123456789/1631

3. Le nombre de manuscrits conservés dans les bibliothèques publiques du monde s'élève au « chiffre très approximatif » de 4000. Leur nombre, dans les cinq pays cités ci-dessus, est respectivement d'environ 1650 aux Pays-Bas, de c. 1000 en Indonésie, de c. 600 en Grande-Bretagne, « un peu moins de » 200 en Malaisie et de c. 130 en France (Chambert-Loir, 1980a, p. 45).

4. Aujourd'hui, l'École des Langues orientales vivantes est connue sous le nom d'Institut national des Langues et Civilisations orientales (INALCO). 
On verra aussi comment plusieurs d'entre eux copiaient des manuscrits conservés dans des bibliothèques européennes, puis ramenaient en France ces copies. Je décrirai et analyserai enfin quelques-uns de ces manuscrits, en mettant l'accent sur ceux conservés à Tournus, et tout particulièrement sur les lettres de Francis Light, qui fonda la colonie britannique de Penang en Malaisie en $1786^{5}$.

\section{Les manuscrits malais en France}

Les manuscrits malais en France ont été répertoriés dans plusieurs catalogues. Ceux de la Bibliothèque nationale à Paris l'ont été pour la première fois dans une liste de 95 manuscrits, publiée par Aristide Marre en 1879. En 1912, Antoine Cabaton publiait un inventaire plus complet des manuscrits malais de cette bibliothèque dans son Catalogue sommaire des manuscrits indiens, indochinois et malayo-polynésiens de la Bibliothèque nationale (Chambert-Loir et Fathurahman, 1999, p. 167) ${ }^{6}$. P. Voorhoeve révisa la partie de ce catalogue concernant les manuscrits malais pour en donner une version plus systématique, plus approfondie et plus complète, qu'il fit paraître dans Archipel 6 en 1973. Environ 130 manuscrits y sont enregistrés et décrits ${ }^{7}$, conservés dans leur grande majorité à Paris, dont le plus grand nombre à la Bibliothèque nationale ${ }^{8}$, mais aussi à Marseille, Rouen et Tournus. Ce nombre ne reflète pas cependant la réalité de celui des œuvres qu'ils contiennent, car très souvent, un manuscrit est en fait une compilation de plusieurs oeuvres (de trois à cinq en ce qui concerne ceux de la Bibliothèque nationale). La Perpustakaan Negara Malaysia (Bibliothèque nationale de Malaisie) a publié en 1991 un " nouveau catalogue » des manuscrits malais en France « beaucoup plus détaillé que les précédents 》 (Chambert-Loir, 1992, p. 315) et « le plus complet » (yang terlengkap) (Chambert-Loir et Fathurahman, 1999, p. 167). Dans ce catalogue, sont enregistrés les manuscrits de la Bibliothèque nationale, des Missions étrangères, des Bibliothèques municipales de Tournus, Marseille et Rouen. Il « comporte » cependant « des omissions et des erreurs qui rendent indispensable le recours à Voorhoeve » (Chambert-Loir, 1992, p. 315). On a également des indications sur les manuscrits malais en France

5. Francis Light était un « ancien officier de la Compagnie anglaise des Indes devenu marchand ». Voulant « donner une base à la Compagnie dans l'île de Pinang, appartenant alors au sultan de Kedah », il ne demanda l'accord ni de la Compagnie, ni du sultan, et « lança une expédition sur l'île dont il prit possession en $1786 »$ (Guillot, 1997, p. 154).

6. Avant la publication du catalogue d'Antoine Cabaton, Édouard Dulaurier avait élaboré un catalogue manuscrit inédit des manuscrits malais de la Bibliothèque nationale, qui fut révisé en 1894, « sous la direction » d'Henri-Léon Feer à laquelle ce dernier ajouta une introduction contenant des explications relatives à la constitution de cette collection, qu'il publia en 1898 , mais ce catalogue révisé resta inédit (Chambert-Loir, 1980a, p. 53), Henri Chambert-loir (1980b, p. 94-98) a publié à nouveau une partie de cette introduction.

7. Chambert-Loir et Fathurahman, 1999, p. 167.

8. Elle en possèderait « un peu plus de $120 »$ (Chambert-Loir, 1980a, p. 52). 
dans le catalogue d'Henri Chambert-Loir et Oman Fathurahman, déjà cité, des collections de manuscrits indonésiens dans le monde ${ }^{9}$.

C'est Paris qui compte le plus grand nombre de manuscrits malais. On y trouve la collection la plus importante de France, à savoir celle de la Bibliothèque nationale. Y sont présentes, par exemple, des œuvres célèbres de la littérature malaise, comme le Bustan al-Salatin (Le jardin des rois), Undang-Undang Melaka (Lois de Malacca), Undang-Undang Laut Melaka (Lois maritimes de Malacca), Adat Raja-Raja (Les coutumes des rois), Hikayat Muhammad Hanafiah (Histoire de Muhammad Hanafiyyah), Hikayat Isma Yatim (Histoire d'Isma Yatim) ou Hikayat Iskandar Zulkarnain (Histoire d'Alexandre le bicornu). Signalons aussi des œuvres plus récentes, bien connues, comme Hikayat Abdullah (Histoire d'Abdullah) et Syair Kampung Gelam Terbakar (Poème de l'incendie de Kampung Gelam) d'Abdullah bin Abdul Kadir Munshi, ou moins connues, comme Syair Dagang Berjual Beli (Poème de l'étranger faisant du commerce) et Syair Potong Gaji (Poème sur la diminution du salaire) de Tuan Simi, et Syair Tengku Perabu (Poème de Tengku Perabu), d'un auteur inconnu. Ces trois derniers poèmes, qui ont fait l'objet d'une édition critique par Muhammad Haji Salleh (1994), entrent dans la catégorie des œuvres de "protestation sociale » (protes sosial) de la part de Malais de Singapour à l'époque de la colonisation anglaise au XIX ${ }^{\mathrm{e}}$ siècle (ibid., p. 32 et 3$)^{10}$.

D'autres institutions de Paris comportent aussi des manuscrits malais.

Le Musée Guimet, en compte une vingtaine ${ }^{11}$. Ils comprennent des œuvres bien connues, comme Hikayat Indera Putera (Histoire d'Indera Putera), Syair Bidasari (Poème de Bidasari), Syair Ken Tambuhan (Poème de Ken Tambuhan), Hikayat Indera Bangsawan (Histoire d'Indera Bangsawan), le Bustan al-Salatin et Hikayat Sultan Ibrahim ibn Adham (Histoire du Sultan Ibrahim ibn Adham). Ils comprennent aussi des œuvres qui le sont moins. Parmi elles, citons celles dans lesquelles il est donné des conseils, comme Hikayat Ikan di dalam Air dengan Raja Gajah (Histoire du poisson dans l'eau et du roi

9. Dans cet ouvrage (p. 167-168), sont également signalés un article de Harun Mat Piah (1982) qui « décrit 5 manuscrits d'histoires de Panji » (memerikan 5 naskah cerita Panji) de la Bibliothèque nationale et un catalogue de 1995 (cf. Katalog 1995) qui " mentionne 7 manuscrits avec la photo de chacun d'eux " (mencatat 7 naskah dengan foto masing-masing) conservés dans cette même bibliothèque. Y sont aussi notés l'ouvrage de Pearson (1971), qui indique la présence de deux manuscrits dans la Bibliothèque municipale de Marseille, un dans celle de Rouen et un dans celle de Tournus, l'article de Claude Guillot (1997), qui parle de quatre manuscrits se trouvant aux Missions étrangères, et l'ouvrage Missions Etrangères et langues orientales... (1997), qui cite six manuscrits présents dans cette institution.

10. Muhammad Haji Salleh a également publié un article les concernant dans Archipel en 1991. Signalons une étude assez récente (Proudfoot, 2001), du manuscrit de cette bibliothèque coté Mal.-pol. 70, « the oldest known manuscript of Hikayat Pelanduk Jenaka » (ibid.).

11. Ces informations sur les manuscrits malais au Musée Guimet proviennent de celles données par P. Voorhoeve (1973, p. 68-73). 
des éléphants) (Mal.-pol. 266), que l'on ne trouve pas ailleurs. Citons aussi, dans le manuscrit Mal.-pol. 274, deux syair, rédigés dans un jawi artistique, intitulés Syair Orang Dagang di Selangor (Poème de l'étranger à Selangor) et Syair Belalang dan Kumbang (Poème de la sauterelle et du bourdon) ${ }^{12}$.

Aux Archives des Missions étrangères, sont conservés aussi quelques manuscrits ${ }^{13}$. J'ai eu l'occasion d'en consulter deux, à savoir une histoire de Panji (MS 1080) et Hikayat Pelanduk Jenaka (Histoire du cerf-nain drôle) (MS 1085). Le premier est daté de 1820 et a été copié par Syeikh Ali al-Din. Le deuxième, originaire de Malacca, est daté de 1237 de l'hégire (soit 1828 ère chrétienne), il fait $19,5 \mathrm{~cm} \times 14,5 \mathrm{~cm}$ et a 78 pages, écrites avec de l'encre noire et rouge. Les manuscrits des Missions étrangères ont probablement été rapportés à Paris par des prêtres ayant séjourné en Malaisie ${ }^{14}$.

On trouve, toujours à Paris, un manuscrit à l'Institut national des Langues et Civilisations orientales (INALCO), à savoir une lettre de Raja Dompu (Sumbawa), datée de 1307 de l'hégire (soit 1889 ère chrétienne) (ChambertLoir et Fathurahman, 1999, p. 168), que je n'ai pas consulté.

La Société asiatique a, pour sa part, abrité un manuscrit malais, intitulé Teromba Pusaka, édité et analysé par Édouard Dulaurier en 1845. Cependant, de nos jours, on a perdu la trace de ce manuscrit (ibid.).

Hors de Paris, les manuscrits malais sont beaucoup moins nombreux, et se trouvent dans les Bibliothèques municipales de Marseille (de 2 à 4 , selon les auteurs) (Chambert-Loir et Fathurahman, 1999, p. 167), de Rouen (1 ou 2, selon les auteurs) (ibid., p. 168) et de Tournus (1 à 5, selon les auteurs) (ibid.), dont j'étudierai le fonds plus loin.

On ne sait pas grand-chose de la valeur réelle de la grande majorité de ces manuscrits malais conservés en France, car ils ont été relativement peu étudiés, aussi bien du point de vue de leur contenu, que de l'authenticité des textes, des auteurs et des copistes.

12. Lorsque j'ai demandé à consulter quelques-uns de ces manuscrits, qui selon le catalogue de P. Voorhoeve (ibid.) constituent un « Dépôt du Musée Guimet », on m'a dit qu'ils étaient conservés au Musée Guimet. Lors de ma visite à la bibliothèque de ce Musée, je n'ai pas pu consulter ces manuscrits, car, selon le bibliothécaire, Monsieur Aroquiadasse Adeikalam, les données relatives à leur localisation sont incomplètes. Je n'ai donc pu les voir que sous forme de microfilms.

13. Leur nombre varie de 2 à 6 selon les auteurs (Chambert-Loir et Fathurahman, 1999, p. 168).

14. R. P. R. Cardon et J. B. Boucho pourraient faire partie de ces prêtres. En effet, dans ces mêmes Archives des Missions étrangères, se trouve un ouvrage intitulé Le Roman de pelandok le chevrotain, publié en 1935 à Bruges en Belgique. Il s'agit de la traduction française de Hikayat Pelanduk Jenaka (sans doute le manuscrit MS 1085 indiqué ci-dessus), réalisée par R. P. R. Cardon, un prêtre ayant séjourné à Penang. On y trouve aussi un ouvrage intitulé Bahua Terteralah Kitab ini di Pulo Pinang dengan izin Terhormati Tuan J. B. Boucho (Ce livre a été imprimé à Penang avec l'autorisation de l'honorable J. B. Boucho), publié à Penang en 1860, relatif à l'enseignement de la religion catholique. Le prêtre Jean-Baptiste Boucho (1797-1871), à lui aussi séjourné à Penang (Launay, 1916, p. 204), où il mourut (http://archives.mepasie.org/ annales-des-missions-etrangeres/le-diocase-de-bayonne-et-la-socia-c-ta-c-des). 


\section{Les enseignants et chercheurs français et les manuscrits malais au XIX ${ }^{\mathrm{e}}$ siècle}

Les premiers enseignants de l'École des Langues orientales vivantes ont été très actifs dans la constitution et le catalogage de la collection des manuscrits malais de la Bibliothèque nationale à Paris. Ils sont également pionniers dans le domaine des études malaises ${ }^{15}$ en France et plusieurs de ces manuscrits leur ont servi de documents pour leurs recherches et leur enseignement. Comme on l'a vu dans la section précédente, les premiers catalogues de cette bibliothèque ont été constitués par Aristide Marre, Édouard Dulaurier et Antoine Cabaton. Certains, tout particulièrement Édouard Dulaurier, et, dans une moindre mesure, l'abbé Pierre Favre, ont copié des manuscrits et/ou ont aidé à leur recueil ${ }^{16}$.

Je donne ci-dessous quelques exemples d'activités scientifiques de ces pionniers de l'enseignement et des études malaises en France à qui il convient de rendre hommage.

Édouard Dulaurier (1807-1881), le premier enseignant de malais à l'École des Langues orientales vivantes, est le " fondateur de la "malayologie française" " (Lombard, 1976, p. 3). Il a copié de nombreux manuscrits conservés dans des bibliothèques européennes (cf. Voorhoeve, 1973). On peut citer la copie d'Hikayat Raja Pasai (Histoire des rois de Pasai) (sans doute le manuscrit de la Bibliothèque nationale de France, coté Mal.-pol. 50), faite à partir du manuscrit en caractères arabes (jawi), datant de 1815, de la Royal Asiatic Society (RAS) de Londres (Raffles Malay 67). C'est au cours d'un voyage dans cette ville en 1838 qu'il réalisa cette copie, qu'il publia en 1849, constituant ainsi la première édition d'Hikayat Raja Pasai (Histoire 2004 et Zaini Lajoubert 2008). Parmi ses autres copies de manuscrits, on peut citer celles d'Undang-Undang Melaka et de diverses autres lois et coutumes malaises, d'Hikayat Raja Banjar dan Kotaringin (Histoire des rois de Banjar et de Kotaringin), de Syair Perang Inggeris di Betawi (Poème de la guerre des Anglais à Batavia), Syair Selindung Delima (Poème de Selindung Delima), Syair Ken Tambuhan, Syair Bidasari, Hikayat Hatim Tai (Histoire de Hatim Tai), Hikayat Si Miskin (Histoire du pauvre), Hikayat Indera Putera, Hikayat Tuan Puteri Jauhar Manikam (Histoire de la princesse Jauhar Manikam), Hikayat Pencuri (Histoire du voleur), de Syair Dagang Berjual Beli, de lettres relatives à Francis Light et de quelques autres œuvres encore. La copie

\footnotetext{
15. Dans cet article, on entend par « études malaises », aussi bien celles relatives à la Malaisie qu'à l'Indonésie. Remarquons qu'en France, les chercheurs sont généralement spécialisés, soit dans les premières, soit dans les secondes.

16. Dans le catalogue de P. Voorhoeve (1973), le nom d'Édouard Dulaurier est en rapport avec 24 manuscrits et celui de l'abbé Pierre Favre avec 5 manuscrits. Notons qu'Henri-Léon Feer (1830-1902), auteur de l'introduction de 1898 (cf. op. cit., note 6), avait été lui aussi un enseignant de l'Ecole nationale des Langues orientales vivantes, non pas de malais, mais de tibétain. Employé ensuite à la Bibliothèque nationale à Paris, il y termina « sa carrière comme conservateur adjoint au département des manuscrits orientaux » (Le Calloc'h, 1995, p. 270).
} 
de manuscrits malais en jawi, comme le faisait surtout Édouard Dulaurier, était alors quelque chose d'assez exceptionnel, car les chercheurs européens utilisaient généralement les services d'écrivains ou de copistes malais pour faire ce travail compliqué, prenant beaucoup de temps. Édouard Dulaurier a même publié en 1843 un ouvrage dans lequel il explique son travail de recueil et de copie de manuscrits malais, tels que ceux provenant de collections de manuscrits conservées à Londres.

L'abbé Pierre Favre (1812-1887), le deuxième enseignant de malais à l'École des Langues orientales vivantes, a « forgé les instruments dont elle se servit pendant de longues années » (Lombard, 1976, p. 3), et est surtout connu pour son Dictionnaire malais-français (1875) et son Dictionnaire français-malais $(1880)^{17}$. Dans le premier $(1875$, p. xxv-xxvi), il énumère quelques textes de littérature malaise qui lui ont servi de corpus pour réaliser son dictionnaire, ce qui montre qu'il avait connaissance d'une partie des principales œuvres malaises de l'époque. Ces œuvres sont Hikayat Amir Hamzah (Histoire d'Amir Hamzah), Hikayat Bispu Raja (Histoire de Bispu Raja), Hikayat Raja Pasai, Hikayat Abdullah, Hikayat Hang Tuah (Histoire de Hang Tuah), Hikayat Isma Yatim, Hikayat Kalilah dan Daminah (Histoire de Kalilah et de Daminah), Syair Ken Tambuhan, Taj al-Salatin, Hikayat Seri Rama (Histoire de Seri Rama), Syair Bidasari, Hikayat Sultan Abdul Muluk (Histoire du sultan Abdul Muluk), Hikayat Sultan Ibrahim (Histoire du sultan Ibrahim) et Sejarah Melayu (Histoire malaise). L'abbé Pierre Favre est également à l'origine de copies de manuscrits malais (cf. Voorhoeve, 1973), bien qu'en moins grand nombre qu'Édouard Dulaurier. Il est également l'auteur de traductions en français, comme celle de Syair Singapura Terbakar (Poème de l'incendie de Singapour) d'Abdullah bin Abdul Kadir Munshi (cf. Favre, 1883).

Aristide Marre (1823-1918), le troisième enseignant de malais à l'École des Langues orientales vivantes, a traduit en français la copie en jawi d'Hikayat Raja Pasai que publia Édouard Dulaurier en 1849, dont j'ai parlé ci-dessus. Cette traduction, la première de ce texte malais, parut en $1874^{18}$. Aristide Marre a également réalisé des traductions d'autres œuvres malaises, comme celle du Taj al-Salatin (La couronne des rois) parue en 1878, de Sejarah Melayu, parue en 1896, et d'Hikayat Jauhar Manikam (Histoire de Jauhar Manikam), parue en 1897, qu'il réalisa avec Édouard Dulaurier (Cabaton, 1912, p. 225).

17. Voir l'analyse par Pierre Labrousse (1976) des dictionnaires de l'abbé Pierre Favre, qu'il compare à ceux de J. Pijnappel et de R. J. Wilkinson.

18. En 2004 (Histoire), les éditions Anacharsis de Toulouse ont réédité cette traduction présentée et annotée par Monique Zaini-Lajoubert. 
Quant à Antoine Cabaton (1863-1942), le cinquième enseignant ${ }^{19}$ de malais à l'École des Langues orientales vivantes ${ }^{20}$, il a été rattaché pendant plusieurs années à la Bibliothèque nationale, ce qui lui permit de rédiger son catalogue de 1912. Il publia dans la Revue du monde musulman « la partie la plus novatrice de ses œuvres, à savoir des études sociologiques de l'islam et des mouvements nationalistes indonésiens, ainsi qu'une chronique attentive de la recherche sur les Indes néerlandaises » (Labrousse, 1995, p. 238). «L'un de ses ouvrages majeurs » est Les Indes néerlandaises (1910) (ibid., p. 239).

Parmi eux, seuls l'abbé Pierre Favre et Antoine Cabaton ont fait un séjour en Insulinde. L'abbé Pierre Favre est resté en Malaisie, à Penang et à Malacca, pendant dix ans environ (Lombard, 1976, p. 3-4). Antoine Cabaton a effectué une mission de deux ans en Indochine et réalisé pendant cette période « un voyage de reconnaissance » aux Indes néerlandaises (Lombard, 1983, p. 20), limité à Java et au nord de Bali, de quelques mois (d'août à octobre 1899) (Labrousse, 1995, p. 238).

Après avoir donné un aperçu général des manuscrits malais en France et des premiers chercheurs et enseignants français qui ont joué un grand rôle dans la constitution de la collection de la Bibliothèque nationale de France, je vais me consacrer maintenant à certains de ces manuscrits, à savoir les lettres relatives à Français Light. Selon mes recherches, j'ai pu trouver qu'il en existe en France un certain nombre de copies : une centaine, à la Bibliothèque nationale à Paris, et 38 , à la Bibliothèque municipale de Tournus. Il se peut que ces copies aient été réalisées à partir de certaines des 1200 "Light Letters » de la School of Oriental and African Studies (SOAS), qui abrite la plus grande collection de ces lettres.

Je vais donner ci-dessous une brève description de ces « Light Letters", puis me concentrer tout particulièrement sur celles de Paris, mais surtout sur celles de Tournus.

\section{Les lettres relatives à Francis Light}

\section{À Londres}

Dans un article paru en 1987 dans Indonesia Circle ${ }^{21}$, Ulrich Kratz donne pour la première fois des informations sur les «Light Letters », conservées à

19. Le quatrième enseignant est Alfred Tugault (1825-1912). Il a eu un rôle beaucoup moins important que les quatre enseignants cités ici, pour ce qui est des premiers développements des études malaises en France. Voir la notice le concernant par Pierre Labrousse (1995, p. 236-237).

20. Pour de plus amples informations sur ces pionniers des études malaises en France, voir " DULAURIER, Édouard », "FAVRE, Pierre, Étienne, Lazare », " MARRE DE MARIN, Aristide », "CABATON, Antoine », (Labrousse, 1995, p. 232-234, 234-235, 235-236, 237239). Sur Pierre Favre, voir aussi Denys Lombard (1976, p 3-8).

21. À partir de 1997, la revue a pris le nom d'Indonesia and the Malay World. 
la SOAS de Londres. Ces lettres sont réparties dans onze fascicules bleus de dimensions diverses et bien entretenus, ayant pour cote MS 40320. Elles ne sont cependant pas encore arrangées de façon systématique, mais, au contraire, mélangées dans ces onze fascicules.

On y trouve, entre autres, de très précieuses informations concernant les rapports entre Francis Light et les souverains malais, dans les domaines diplomatiques, commerciaux, relatifs aux relations conflictuelles et amicales, aux aides financières, à la sécurité, etc.

Ulrich Kratz explique que c'est William Marsden (1754-1836), qui a été employé de l'East India Company à Sumatra (vers 1771-1779), qui a fait don de ces lettres à la bibliothèque du King's College de Londres en 1835. À la fondation de la SOAS en 1916 (qui s'appelait alors School of Oriental Studies), elles ont été tranférées à la bibliothèque de cette institution, où elles se trouvent encore aujourd'hui. On ne connaissait pratiquement rien du contenu de ces lettres avant la parution de l'article d'Ulrich Kratz qui les étudie, en mettant tout particulièrement l'accent sur les relations commerciales de Francis Light avec les souverains malais. Il classe ces «Light Letters » en cinq catégories :

1. Letters sent to Light;

2. Letters sent to Light's business partner Scott;

3. Originals, copies and drafts of letters sent by Light ;

4. Internal Malay correspondence;

5. Other documents (bills, receipts, petitions, business contracts).

Si les quatre premières catégories sont les «Light Letters » proprement dites, la dernière ( Other documents ») n'en comprend pas moins des notes importantes sur la Penang d'autrefois, comme le texte de l'accord de 1791 entre Sayid Hussain Aidid et Francis Light. À signaler aussi une Babat Suku Orang Kelang yang Anak Bini dalam Pekan Pulau Pinang (Chronique d'une personne de la communauté de Kelang dont l'épouse se trouve dans la ville de l'île de Pinang), contenant une liste de noms des commerçants vivant à Penang, avec le nombre de boutiques, de membres de leur famille et de leurs esclaves.

Ces « Light Letters 》 sont très importantes pour l'histoire de Penang, tout particulièrement vers 1786-1794. Des recherches approfondies sur l'ensemble de ces lettres s'imposent donc. Elles demanderont cependant beaucoup de temps et d'efforts, d'autant plus que, comme nous l'avons vu plus haut, elles ne sont pas encore classées de façon systématique.

\section{À Paris}

Aucun des catalogues recensant les manuscrits conservés à Paris, dont j'ai parlé ci-dessus, ne mentionne la présence de lettres relatives à Francis Light. Lors de ma visite à la Bibliothèque nationale de France, j'ai pu constater que ces lettres étaient rassemblées dans le manuscrit coté Mal.-pol. 97. 
Au sujet de ce manuscrit, P. Voorhoeve (1973, p. 59), parle d'une collection de lettres diverses, pas seulement malaises, mais aussi javanaises et bugis, et donne quelques informations sommaires les concernant. Voici comment il décrit ce manuscrit :

"Collection de lettres javanaises, bougui et malayes, provenant des portefeuilles de Marsden, de ceux de l'Académie de Delft et de différentes communications particulières." Copiées par Dulaurier. - Quelques lettres sont des originaux, un certain nombre sont autographiées. $\mathrm{XIX}^{\mathrm{e}}$ siècle. Écritures neskhi, javanaise, latine et (p. 202/203, datées 1798/1799) bugis, $380 \times 240 \mathrm{~mm}, 408$ pages, nombre de lignes variable. Demi-rel. chagrin. Ancien numéro : Malais-Javanais 60.

Le catalogue de la Bibliothèque nationale de Malaisie (1991, p. 98) ne donne pas dans l'ensemble les mêmes informations au sujet de ce manuscrit. Il parle, par exemple, du type de papier, de son filigrane, de l'encre utilisée, de la reliure. Il cite deux destinataires de lettres : le " Commander Charles Med di negeri Malbara» (commandant Charles Med à Malbara (sans doute Malabar)) et "Jan Corpes yang memerintah pesisir barat Padang 》 (Jan Corpes, qui gouverne la côte ouest de Padang). Il indique que l'on trouve aussi " pelbagai surat dari Sultan Melayu, di antaranya Sultan Selangor» (diverses lettres de sultans malais, dont celui de Selangor) et que ces lettres ont été écrites entre 1781-1787. Il parle du copiste, mais contrairement au catalogue de P. Voorhoeve, en des termes vagues: "surat yang ditulis oleh seorang jurutulis sebagai contoh surat-surat » (lettres écrites par un copiste comme exemple de lettres).

Dans le passage consacré à la collection de manuscrits de la Bibliothèque nationale, dans Henri Chambert-Loir et Oman Fathurahman (p. 167-168), il n'est pas non plus fait mention de la présence de lettres relatives à Francis Light parmi ces manuscrits.

Selon mes recherches, le nombre de lettres, en grande majorité se rapportant à Francis Light, du manuscrit Mal-pol 97, s'élève à une centaine. Comme indiqué dans le catalogue de P. Voorhoeve, ce sont des copies, réalisées par Édouard Dulaurier. Ces lettres, échangées la plupart du temps avec des souverains et des hauts personnages malais, concernent diverses affaires de Francis Light quand il se trouvait à Penang. Dans la plupart d'entre elles, Francis Light est désigné par « Gurnadur 》 (Gouverneur) ou «Sinyor Gurnadur » (Monsieur le Gouverneur).

Mises à part les lettres relatives à Francis Light, on trouve aussi dans ce manuscrit des lettres de plusieurs fonctionnaires anglais à Sumatra (de Fort Malborough, à Bengkulu) échangées avec des souverains et des hauts personnages malais de Moko-Moko, de Bengkulu, d'Andalas et de Padang, ainsi qu'une lettre du Sultan Alauddin Mansur Shah d'Aceh au «Raja Perancis yang nama Louis Phillip » (Roi français du nom de Louis Philippe).

On ne sait pas très bien pourquoi Édouard Dulaurier a copié ces lettres, car aucun de ses écrits connus ne parlent explicitement de ces dernières. Je 
ne peux que supposer qu'il aurait fait ces copies, dans les années 1840, à une époque ou il copiait activement des manuscrits malais conservés à Londres, qu'il rapportait ensuite à Paris pour enrichir les documents historiques sur le monde malais, et pour les utiliser pour l'enseignement à l'École des Langues orientales vivantes. On ne sait pas non plus s'il a vraiment réalisé ses copies à partir de lettres conservées à la SOAS de Londres (MS 40320), dont je viens de parler ci-dessus. Seules des recherches plus approfondies sur les «Light Letters » permettront de répondre à cette question. Un indice cependant nous indique que tel pourrait être le cas. En effet, comme on l'a vu plus haut (p. 6), c'est William Marsden qui a fait don des «Light Letters » conservées à la SOAS. Or, dans sa description du manuscrit Mal-pol 97 de la Bibliothèque nationale de France, où se trouvent les lettres relatives à Francis Light, P. Voorhoeve (1973, p. 59), parle d'une collection de lettres diverses, « provenant », entre autres, « des portefeuilles de Marsden » (cf. op. cit., p. 7).

\section{À Tournus}

Parmi les catalogues recensant les manuscrits malais de Tournus, dont j'ai parlé ci-dessus, l'un d'eux indique la présence de deux lettres qui pourraient être relatives à Francis Light, bien que son nom ne soit pas indiqué.

Dans sa description de ces manuscrits, P. Voorhoeve (1973, p. 75) ne fait aucune mention de lettres. Il ne parle que d' " une collection de contes du même genre que ceux qui sont intercalés dans la Hikayat Bakhtiyar », dont le début et la fin manquent et dont il n'a vu que deux fragements, ce qui ne lui permet pas de déterminer le titre. Il indique cependant qu'un « personnage qui joue un rôle dans le premier fragment s'appelle Alkas menteri ».

Le catalogue de la Bibliothèque nationale de Malaisie (1991, p. 178-182) donne plus de détails que celui de P. Voorhoeve, fait mention de l'existence de lettres et indique la présence de trois manuscrits cotés M 29, M 30 et M 31. Selon lui, le M 29 se compose de la Hikayat Amir Hamzah, texte rédigé avec soin de 105 pages, incomplet (le début et la fin manquent), commençant par 1'histoire d'Alqas Menteri qui tue Khoja Bahti, et se terminant par Landuhur, arrêté par le prince. Le M 30 comprend le Syair Ken Tambuhan et des lettres. Le Syair, de 137 pages, a été copié avec minutie le 5 avril 1820, sans doute par un copiste européen. Les lettres, de 7 pages, et copiées par le même copiste que le syair, sont au nombre de trois : une lettre de Datok Kaya Batu Behara au «Sinyor Gornadur » ${ }^{22}$, datée du 4 novembre ; une lettre d'Encik Longok de Perlis au «Kapten Ascot » ${ }^{23}$ à Salang, datée du 17 mars 1785 ; une lettre de Tengku Kajuran Japura de Langkat au «Sinyor Gornadur », à Penang ${ }^{24}$.

22. Il s'agit sans doute de l'une des deux lettres qui pourraient être relatives à Francis Light.

23. Il s'agit sans doute du Capitaine James Scott.

24. Il s'agit sans doute de la deuxième lettre relative à Francis Light (cf. note 22). 
Le M 31 se compose de lettres et de Syair Burung Nuri (Poème de la perruche à collier). Les lettres, de cinq pages, copiées avec soin par un copiste européen, sont également au nombre de trois : une lettre du sultan Ibrahim de Selangor au « Jeneral » au Bengale, datée du 7 décembre 1785 ; une lettre d'un homme à son frère (saudaranya), datée du 16 août 1792 ; une lettre du sultan Maki al-din, Raja Bacan, au " Gurnadur » Arin qui gouverne Ambon. Le syair, complet, de 43 folios, a été copié le 8 août 1827, sans doute par le même copiste européen que le M 30 et les lettres du M 31.

Dans le court passage relatif aux manuscrits de la Bibliothèque municipale de Tournus, dans Henri Chambert-Loir et Oman Fathurahman (p. 168), la présence de lettres relatives à Francis Light parmi ces manuscrits n'est pas indiquée.

Je me suis rendu à la Bibliothèque municipale de Tournus (en région Bourgogne), dont les locaux sont situés dans la cour du cloître de l'Abbaye Saint-Philibert, pour examiner ces trois manuscrits. Ceux-ci sont inventoriés à la page 387 du catalogue manuscrit de cette bibliothèque réalisé en 1886 par Jean Martin, qui en était alors le directeur, sous les cotes indiquées ci-dessus, soit M 29, M 30 et M 31, qui correspondent à trois fascicules, et dont on ne sait pas comment ils sont arrivés à Tournus. Ces manuscrits n'ont pas encore attiré l'attention des chercheurs et n'ont donc pas fait l'objet d'une étude spécifique.

Après avoir examiné ces trois fascicules, $\mathrm{j}$ 'ai pu constater que c'est dans le M 30 que se trouvent les 38 lettres relatives à Francis Light. Avant de me pencher tout particulièrement sur celles-ci, je donnerai une description de ces trois manuscrits, qui j'espère pourront enrichir les informations les concernant enregistrées dans les catalogues existants que j'ai résumées ci-dessus.

Le manuscrit M $29(25 \mathrm{~cm} \times 19 \mathrm{~cm})$, relié avec du papier cartonné, est écrit en jawi avec de l'encre noire et rouge. Il a 105 pages de 22 lignes chacune. Le papier, assez usé, a un filigrane représentant trois croissants de lune. Le manuscrit ne comporte ni introduction, ni colophon. Il contient 17 histoires et récits d'origine musulmane. Ces histoires et récits ont pour but de donner une connaissance et des conseils aux lecteurs sur les bons ou mauvais enseignements qu'il convient de suivre ou ne pas suivre dans la vie, un type de texte que l'on rencontre couramment dans des œuvres comme le Taj al-Salatin ou le Bustan al-Salatin. Le nom du copiste et la date de la copie ne sont pas indiqués.

Le manuscrit M $31(22 \mathrm{~cm} \times 17 \mathrm{~cm})$, comporte 112 pages et est écrit en jawi avec de l'encre noire. Il contient la copie de deux œuvres, le Syair Si Burung Nuri serta Si Bunga Chempaka (Poème de la perruche à collier et de la fleur de frangipanier) (p. 1-52) et Syair Ikan Tambera Berganding sama Kakap (Poème du poisson tambera ${ }^{25}$ côte à côte avec un barramundi) (p. 53-87). Le colophon du premier syair contient la date du 7 août 1728 et celui du deuxième, « Rejab ${ }^{26}$ », année 1235 de l'hégire (soit 1826 ère chrétienne). Notons que

\section{Labeobarbus tambra.}

26. Septième mois de l'hégire. 
la Bibliothèque nationale de France contient également une œuvre intitulée Syair Nuri (Mal-Pol. 92), originaire de Batavia (datée de 1827) (Voorhoeve, 1973, p. 58), et une autre appelée Syair Ikan Tambera (Mal-Pol. 88), une copie réalisée par Édouard Dulaurier, à partir du manuscrit conservé à la RAS (Raffles Malay 7), sans indication de date (ibid., p. 44). On ne sait pas à partir de quels textes ont été copiés les deux syair de la Bibliothèque municipale de Tournus, peut-être à partir de ceux de la Bibliothèque nationale à Paris, ou de ceux conservés à Londres ou ailleurs. À la suite de ces deux syair, on trouve la copie d'une lettre du Sultan Ibrahim de Selangor au gouvernement anglais du Bengale (p. 90-91), d'une lettre de Raja Benchana (p. 93-95), de celle du Sultan Mansur Syah de Terengganu aux Anglais ( $p$ 95-97), et de celle du commerçant Qamar al-Din au Gouverneur (Gurnadur) de Penang (p. 97-100). Il y a également la copie d'un extrait d'une hikayat contenant des éléments hindouistes (p. 100-103). Le manuscrit se termine par plusieurs strophes d'un pantun d'amour (p. 106-112). Le manuscrit M 31 est donc une compilation de plusieurs auvres et de lettres de souverains malais aux caractéristiques diverses. Le style de l'écriture jawi est le même dans tout le manuscrit, ce qui laisse supposer qu'il est l'œuvre d'un seul et même copiste, une personne en tout cas ayant réalisé son travail de copiste avec grand soin, puisqu'on ne relève aucune erreur dans les œuvres ou les lettres qu'il a copiées.

Le manuscrit M 30 de la Bibliothèque municipale de Tournus $(22 \mathrm{~cm} \times 17 \mathrm{~cm})$ comprend 235 pages de 15 lignes chacune. Il est rédigé sur du papier sans filigrane et sa reliure est soignée. Il est écrit avec de l'encre noire, très soigneusement, avec grande attention et d'une manière assez artistique. Le style du jawi est le même dans l'ensemble du manuscrit, ce qui laisse supposer qu'il est l'œuvre d'un seul et même copiste. De même, le style du jawi du manuscrit M 31 est semblable à celui du manuscrit M 30 et il y a fort à parier que ces deux manuscrits aient été copiés par la même personne.

Le manuscrit M 30 comprend deux parties. La première se compose de la copie d'un syair de 137 pages, le Syair Ken Tambuhan. Il comprend une introduction et un colophon, daté du 20 Jamadil $^{27}$, fin de l'année 1235 de l'hégire (soit 1826 ère chrétienne). Le nom du copiste et la provenance du syair ne sont pas indiqués. La Bibliothèque nationale de France possède deux copies du Syair Ken Tambuhan réalisées par Édouard Dulaurier, l'une (Mal.-pol. 71) à partir du texte de la SOAS (12914B), l'autre (Mal.-pol. 72), à partir de celui de la RAS (Raffles Malay 7) (Voorhoeve, 1973, p. 43-44). Il est probable que le manuscrit M 30 ait été copié d'après l'une des deux copies conservées à Paris ou même directement d'après le texte se trouvant à Londres.

La deuxième partie du manuscrit M 30 est celle qui se rapporte à Penang, et à Francis Light en particulier. Elle contient en effet 38 copies de lettres échangées entre Francis Light et des souverains, ainsi que des hauts

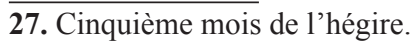


personnages malais. Cette partie commence par le titre suivant : "Inilah segala surat dari Tanah Melayu » (Voici toutes les lettres du Pays malais) (p. 148). Ces lettres sont copiées avec soin et attention, sans aucune faute d'orthographe ou de grammaire. Le copiste n'indique pas leur origine. D'après mes recherches, l'ensemble de ces 38 copies de lettres relatives à Francis Light ressemble beaucoup à celles regroupées dans le manuscrit coté Mal.-pol. 97 de la Bibliothèque nationale à Paris. J'aurais même tendance à dire que la copie des lettres relatives à Francis Light du manuscrit M 30 de Tournus a été faite d'après celles de Paris. Il est intéressant en tout cas de constater qu'une personne, dont on ne connaît pas l'identité, consciente de l'importance des lettres concernant Francis Light, a jugé bon d'en copier quelques-unes. Je donne ci-dessous la liste, avec un résumé succinct, de ces 38 lettres :28

1. p. $152-155^{28}$ : Lettre de Datu Kaya Batu Bahara au Gouverneur (Gurnadur) en charge du gouvernement de Penang (Bandar Pulau Pinang).

2. p. 155-157: Lettre d'Encik Longok, résidant à Bandar Perlis Kota Indera Kayangan Negeri Kedah Dar al-Aman, au Capitaine James Scott (Kapitan Askat) de Salang (Negeri Salang). Lettre datée de 1199 de l'hégire (soit 1788 ère chrétienne).

3. p. 157-159: Lettre de Tengku Kejuruan Muda, résidant à Langkat (Negeri Langkat), au Gouverneur (Gurnadur) résidant à Penang (Negeri Pulau Pinang).

4. p. 159-161 : Lettre de Raja Long Omar de Kedah Dar al-Aman au « Datuk Gurnadur » sage et avisé, qui exerce le pouvoir à Penang (Bandar Pulau Pinang). Dans cette lettre, il est demandé à Francis Light de se pencher sur le conflit commercial entre deux commerçants de Penang (Pulau Pinang) (cf. Annexe 3).

5. p. 162-163: Lettre du souverain de Selangor au Capitaine Thomas Forrest, lui demandant d'aider Selangor à établir des relations amicales et commerciales avec les Anglais au Bengale (en Inde).

6. p. 163-165: Lettre de Francis Light au Roi de Pidir (Tengku Raja Pidir), datée de 1203 de l'hégire (soit 1792 ère chrétienne), relative à l'escroquerie financière d'un capitaine néerlandais (cf. Annexe 1).

7. p. 165-167: Lettre d'allégeance (Surat sembah) du commerçant Dato' Marikan Kandu, dans laquelle il demande à Francis Light de mettre un terme à son différend avec un commerçant de Kelang (cf. Annexe 2).

8. p. 168-169: Lettre de Datuk Paduka Seri Indera, chef (penghulu) de Lima Laras, au « Gurnadur» de Penang (Pulau Pinang).

9. p. 170-171: Lettre d'Encik Piah de Selangor à la fille du « Gurnadur » de Penang (Pulau Pinang).

10. p. 172-173 : Lettre de Datuk Bayan au « Gurnadur » de Penang (Pulau Pinang).

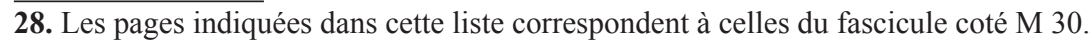


11. p. 174-176: Lettre du prétendant au trône (Raja Muda) de Perlis Indera Kayangan au « Gurnadur » de Penang (Pulau Pinang) concernant le commerce du soufre et demandant que soient réparées trois montres défectueuses.

12. p. 176-177: Lettre de Datu Kaya Batu Bahara au «Gurnadur» de Penang (Pulau Pinang).

13. p. 178-179: Lettre de Mahmud au « Gurnadur» de Penang (Pulau Pinang).

14. p. 180-181 : Lettre de Datu Batu Bahara au « Gurnadur» de Penang (Pulau Pinang).

15. p. 182-184: Lettre de l'Amiral (Laksamana) au «Gurnadur» de Penang (Pulau Pinang).

16. p. 185-187: Lettre du Chef du Port (Syahbandar) de Selangor à Monsieur le Gouverneur Light (Sinyur Gurnadur Mister Light), datée 1196 de l'hégire (soit 1786 ère chrétienne), concernant le commerce du tabac et du tissu à Penang (Pulau Pinang).

17. p.187-189: Lettre du noble (Orang Kaya) Buluh China au « Gurnadur » de Penang (Pulau Pinang).

18. p. 190-191 : Lettre de l'épouse du prétendant au trône (Tengku Raja Muda), représentant du Sultan Ibrahim Syah, en charge du gouvernement de Selangor (Bandar Negeri Selangor), au Gurnadur de Penang (Pulau Pinang).

19. p. 191-193 : Lettre au « Gurnadur» de Penang (Pulau Pinang) au sujet des bateaux se rendant en Arabie.

20. p. 194-195 : Lettre de Tengku Langkat au « Gurnadur » de Penang (Pulau Pinang).

21. p. 196-197: Lettre au sujet du commerce de l'étain.

22. p. 198-200: Lettre de Salang au Capitaine James Scott (Kapitan Askat).

23. p. 201-203 : Lettre d'un commerçant de Kelantan au juge (hakim Bandar) en poste sous l'autorité du « Gurnadur» à Penang (Pulau Pinang). Dans le colophon, il est noté : « Ini surat daripada rahsia raja dari Negeri Kelantan » (Ceci est une lettre confidentielle du souverain de Kelantan).

24. p. 203-205: Lettre du souverain (Paduka Maharaja) de Kedah au « Gurnadur» de Penang (Pulau Pinang).

25. p. 206-208: Lettre du Prince (Pangeran) de Palembang au « Gurnadur » de Penang (Pulau Pinang).

26. p. 208-210 : Lettre de Raja Ali au « Gurnadur» de Penang (Pulau Pinang) écrite à Selangor.

27. p 210-212: $\quad$ Lettre du souverain de Selangor Badar al-Din au « Gurnadur » de Penang (Pulau Pinang) concernant des dettes.

28. p 212-214 : Lettre du Sultan de Selangor à Monsieur le Gouverneur (Sinyor Gurnadur) de Penang (Pulau Pinang), datée de 1201 de l'hégire (soit 1790 ère chrétienne), sur le fait que Sa Majesté désire rencontrer Francis Light pour parler de choses concernant le commerce.

29. p. 214-215 : Lettre de Francis Light au commerçant (Tok Saudagar) Nasar al-Din dans laquelle il lui est demandé d'envoyer de l'étain et du poivre à Penang (Pulau Pinang). 
30. p. 215-216: Lettre de Francis Light à « Tengku Raja Pidir », concernant des affaires commerciales (cf. Annexe 4).

31. p. 217-219: Lettre de Long Ismail au « Gurnadur » de Penang (Pulau Pinang).

32. p. 219-221 : Lettre de Batu Bahara au « Gurnadur» de Penang (Pulau Pinang).

33. p. 221-222: Lettre de Langat au « Gurnadur» de Penang (Pulau Pinang).

34. p. 222-225: Lettre du commerçant secret (saudagar rahsia) du souverain de Kelantan au « Gurnadur » de Penang (Pulau Pinang).

35. p. 225-228: Lettre du commerçant (Datuk saudagar) Nasar al-Din au « Gurnadur » de Penang (Pulau Pinang).

36. p. 228-230: Lettre d'Encik Khamas de Johor au « Gurnadur» de Penang (Pulau Pinang).

37. p. 230-233: Lettre du Chef du Port (Syahbandar), représentant du sultan, au « Gurnadur » de Penang (Pulau Pinang).

38. p. 234-235: Lettre de l'Amiral (Laksamana) de Perak au « Gurnadur » de Penang (Pulau Pinang), datée de 1206 de l'hégire (soit 1794, ère chrétienne).

La liste des lettres ci-dessus permet d'avoir quelques informations sur les relations entre Francis Light et des souverains, ainsi que des hauts personnages malais, surtout pour ce qui est des États de Selangor, Kedah, Perak, Kelantan, Johor et de quelques sultanats malais de Sumatra, comme Langkat, Batu Bahara et Palembang. Ces lettres concernent diverses questions commerciales, des demandes d'aide financière, des conflits commerciaux, mais aussi des choses plus amicales et personnelles. Bien que la colonisation anglaise de Penang soit alors encore récente, Francis Light exerçait déjà, semble-t-il, une grande influence sur des souverains malais, comme on le voit par les compliments dont il fait l'objet de la part de ces derniers et de hauts personnages malais. On en a un exemple dans l'introduction de la lettre de Raja Long Omar de Kedah à Francis Light (cf. la liste, p. 159-160), dont je donne ci-dessous la translittération en caractères latins et sa traduction en français (cf. l'original en jawi dans l'Annexe 3) :

\section{Al-Mustahaq}

Omar ibn Muhammad Taha

Surat kasih sayang tulus ikhlas daripada beta Raja Long Omar di Negeri Kedah Dar al-Aman sampai pada sahabat beta Dato' Gurnadur yang arif bijaksana dan yang mempunyai perintah di dalam Bandar Pulau Pinang serta yang amat mengasihani segala hamba Allah yang teraniaya supaya jadi ramailah segala dagang masuk berniaga ke dalam Pulau Pinang itu dengan sebab baik bicara dan tuah sahabat beta jadi makmurlah di dalam Bandar Pulau Pinang itu. 


\section{Traduction :}

Al-Mustahaq ${ }^{29}$

Omar ibn Muhammad Taha

Lettre d'affection et de dévouement, de moi, Raja Long Omar, de Kedah, pour mon ami, le Gouverneur sage et avisé, en charge du gouvernement de Penang, ayant un grand attachement pour tous les sujets persécutés d'Allah, souhaitant que les étrangers viennent commercer en grand nombre à Penang, car grâce à la sagacité et à la félicité de mon ami, Penang est prospère.

Comme on le voit, ces lettres sont pour la plupart adressées à Francis Light. Trois cependant sont des lettres de Francis Light destinées à des souverains ou des hauts personnages malais. Il s'agit de deux lettres pour Tengku Raja Pidir (cf. la liste, p. 163-165 et p. 215-216) (cf. Annexes 1 et 4) et d'une lettre pour le commerçant (Tok Saudagar) Nasar al-Din (cf. la liste, p. 214-215). Ces trois lettres montrent une convention d'écriture de la correspondance assez homogène, comprenant des paroles d'introduction agréables, chargées de compliments, suivies des propos de la lettre, puis des formules de politesse de fin de lettre, et enfin de la date. Il est sûr que Francis Light avait à son service un secrétaire expérimenté, l'aidant à traiter avec des souverains et de hauts personnages malais.

On en a un exemple notable dans la lettre de Francis Light à Tengku Raja Pidir (cf. la liste, p. 163-165), dans laquelle ce dernier est complimenté, recevant le qualificatif de personne avisée et célèbre. Quand au contenu de la lettre, il concerne un capitaine néerlandais ayant escroqué Francis Light de 368 riyal, qui auraient dû être remis à Tengku Raja Pidir. La manœuvre de Francis Light est claire. Il promet de dédommager Tengku Raja Pidir et de condamner le capitaine néerlandais. En contrepartie, il demande à Tengku Raja Pidir d'aider le capitaine James Scott (Kapitan Askat) dans les affaires qu'il est en train de mener à Pidir. Cette lettre a certainement donné entière satisfaction à Tengku Raja Pidir, qui accorda tout de suite sa coopération pour ce qui est des affaires politiques et commerciales menées entre Pidir et Penang. La lettre date de 1203 de l'hégire (soit 1792 ère chrétienne), c'est-à-dire environ six ans après l'arrivée de Francis Light à Penang.

Dans cette collection de copies de lettres conservées à la Bibliothèque municipale de Tournus, on trouve aussi une lettre assez différente des autres pour ce qui est de sa rédaction et de son intention (cf. la liste, p. 165-167). L'expéditeur est un certain Marikan Kandu et elle est adressée à « qadam ${ }^{30}$ Dato' Gurnadur ». Il s'agit en fait de Francis Light, mais son nom n'est pas mentionné (cf. Annexe 2). Dans cette lettre, Marikan Kandu se plaint d'une personne de Kelang, qu'il qualifie de « fakir Saba », qui l'a escroqué, qui

29. Mot généralement placé en tête de lettre pour indiquer que c'est une lettre «mustahak » (importante).

30. Sans doute kadam («Ar. Sole of foot...a form of adress to royalty ») (R.J. Wilkinson 1959). 
refuse de payer ses dettes s'élevant à 210 riyal, malgré l'injonction du tribunal. Cette lettre non datée montre que les conflits commerciaux survenant à Penang devaient être réglés par l'intermédiaire du tribunal anglais. Il est assez intéressant de constater que le nom de l'expéditeur de la lettre, Marikan Kandu, donne une information importante, à savoir la présence de descendants de « Merican » ou de « Marican $»^{31}$ peu après la fondation de Penang. Le style de la lettre et le malais utilisés sont assez peu soignés, contrairement aux lettres de souverains ou de hauts personnages malais.

Les manuscrits malais en France sont enregistrés et décrits dans des catalogues, qui ne rendent pas toujours compte de leur nombre et de leur contenu réels. On le voit par exemple avec les lettres relatives à Francis Light que j'ai étudiées plus particulièrement dans cet article. En effet, même si certains indices laissent supposer qu'il s'agit de lettres le concernant, on ne peut en avoir la certitude qu'en allant sur place examiner ces manuscrits. Il en est de même pour s'assurer de leur nombre. Grâce à la mission de recherche que j'ai effectuée en France en juillet 2011, j'ai pu avoir une idée plus précise du nombre, de la forme et du contenu des manuscrits malais, en particulier des lettres relatives à Francis Light, conservés en France, tout particulièrement à Paris, mais surtout à Tournus. J'ai ainsi trouvé que la Bibliothèque nationale de France abritait une centaine de lettres le concernant et que la Bibliothèque municipale de Tournus en contenait 38. Je me suis surtout penché sur ces dernières, j'en ai dressé la liste, avec un résumé succinct, et fait l'analyse de certaines. J'ai également donné en annexe la translittération en caractères latins de deux de ces lettres et l'introduction de deux autres sous leur forme originelle en jawi. Les manuscrits malais en France, y compris les lettres relatives à Francis Light, sont encore largement inexplorés. Des recherches plus approfondies pourraient nous réserver certainement encore d'autres surprises et nous permettre ainsi d'enrichir l'histoire des études de la langue et de la littérature malaises en général et celle de Penang et de ses relations avec la France en particulier.

31. Selon R.J. Wilkinson (ibid.), Marikan ou Merikan signifie «A sort of title borne by Tamil Moslems of good position » ou «American ». 


\section{ANNEXES}

\section{Annexe 1}

Translittération en caractères latins de la lettre en jawi de Francis Light à Tengku Raja Pidir [Source : Fascicule coté M 30, Bibliothèque municipale de Tournus, p. 163-165]

\section{Al-qamr wa al-syams}

Surattulus ikhlas muafakatyang tiada berkesudahan selagi ada chakrawala matahari dan bulan daripada beta Gurnadur yang memerintahkan di kota Bandar Pulau Pinang, barang disampaikan Tuhan seru alam apalah kiranya kepada pihak sahabat beta Tengku Raja Pidir yang aqil bijaksana, telah masyhur gah kepujian sampai ke mana-mana.

Wa ba'du dari itu ahwal akan surat sahabat beta dibawa kerani itu sampailah kepada beta dengan sempurnanya. Maka akan perintah piutang Kapitan Belanda pada sahabat beta itu maka sudah beta periksanya akunya tiga ratus enam puluh dulapan riyal itu kepada sahabat beta serta dengan surat beta pun dibawanya sama pergi. Maka ini khabarnya ia sudah pergi ke Benggala.

Maka pasal riyal tiga ratus enam puluh dulapan riyal seperti akuan Kapitan Belanda itu betalah ganti bayar. Apabila ia datang dari Benggala boleh beta kerasi ambil riyal itu kerana ditipunya beta hendak pergi bayar kepada sahabat beta.

Sebermula Kapitan Askat pun beta suruh pergi ke Pidir, itu pun minta sahabat beta tolong barang sesuatu hal-ahwal mereka itu, boleh balik dengan segeranya. Amin.

Perbuat surat ini pada dua hari timbul bulan Rejab hari Ahad sanat 1203 tahun Ba. Tamat. 


\section{Annexe 2}

\section{Translittération en caractères latins de la lettre en jawi de Marikan} Kandu à Francis Light [Source : Fascicule coté M 30, Bibliothèque municipale de Tournus, p. 165-167]

\section{Ya Aziz}

Ampun hamba Dato', Marikan Kandu sakai hamba yang teramat hina maklumkan sembah pada ke bawah qadam Dato' Gurnadur yang teramat mulia. Hamba Dato' maklumkan ahwal hal diri hamba Dato' duduk selama makan pagi sekalian anak-beranak hamba Dato' pun dengan 'asar rahim qadam Dato'.

Di dalam antara itu datang fakir Saba dari Negeri Kelang tanya pada hamba Dato' andak duduk dengan hamba Dato', maka modalnya pun tiada. Kemudian hamba Dato' kasihan sebab dia orang tiada modal, hamba Dato' ajak tiarah di dalam kedai, sekalian sambut-menyambut pada orang kapal atau kepada orang di negeri pun hamba Dato'seorang. Maka fakir Saba itu duduk di kedai sahaja.

Kemudian ia antar pergi ke Pidir dua ratus riyal pun dagangan kain-kain di kedai itu pun tiada ia beri tahu kepada hamba Dato', orang datang kata kepada hamba Dato', kemudian hamba Dato'tahu. Dan lagi beberapa pulak ia antar pergi ke Negeri Kelang itu pun tiada hamba Dato' tahu, kemudian hamba Dato'tahu.

Budi perangai fakir Saba hamba Dato' ajak selesaikan daftar berhadapan saudagar Banna Saba dan berhadapan Mira Lebai, selesai sekalian daftar tinggal lagi baki atas fakir Saba dua ratus sepuluh riyal. Maka sekarang ia pulak tengah duduk kiranya daftar banyak sangat pulasnya.

Sebab pun hamba Dato' teriak kepada qadam Dato' suruh selesaikan berhadapan nakhoda kapal Kelang itu pun Takdur Husin pun mau panggil sekalian nakhoda itu. Sebab pun hamba Dato' datang mari teriak pada ke bawah qadam Dato'jika dengan benar qadam Dato 'pinta qadam Dato'suruh pada nakhoda kapal Kelang selesaikan daftar itu, kemudian boleh qadam Dato 'tahu ia kepada salah benar hamba Dato'.

Adapun duduk hamba Dato' anak-beranak melainkan harap kepada qadam Dato'. Ahwal inilah hamba Dato' maklumkan pada ke bawah qadam Dato'. Tamat. 


\section{Annexe 3}

Introduction de la lettre de Raja Long Omar à Francis Light [Source : Fascicule coté M 30, Bibliothèque municipale de Tournus, p. 159-160] (cf. sa translittération en caractères latins et sa traduction française, op. cit., p. 12 et 13).
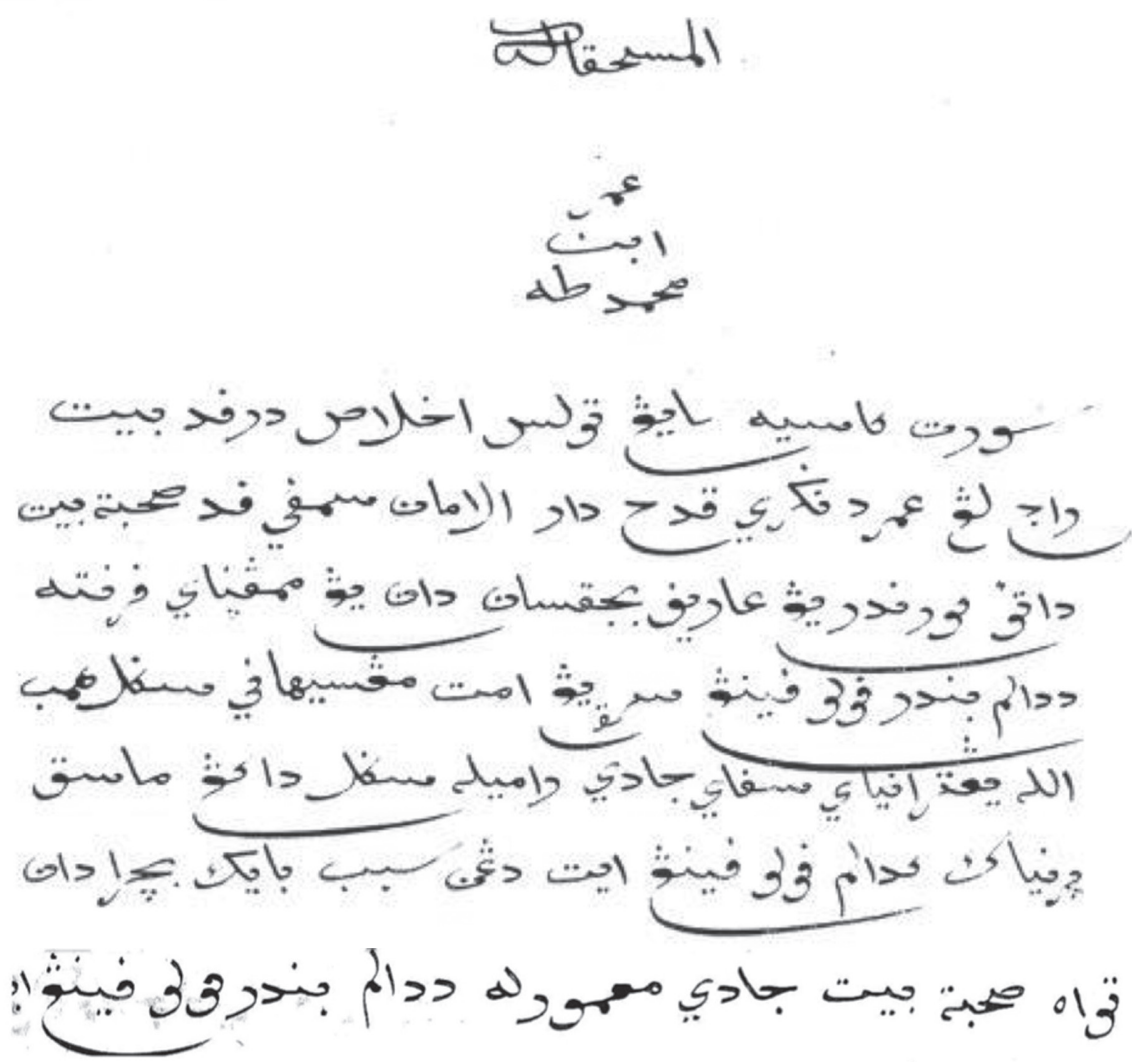


\section{Annexe 4}

Introduction de la lettre de Francis Light à Tengku Raja Pidir [Source : Fascicule coté M 30, Bibliothèque municipale de Tournus, p. 215]
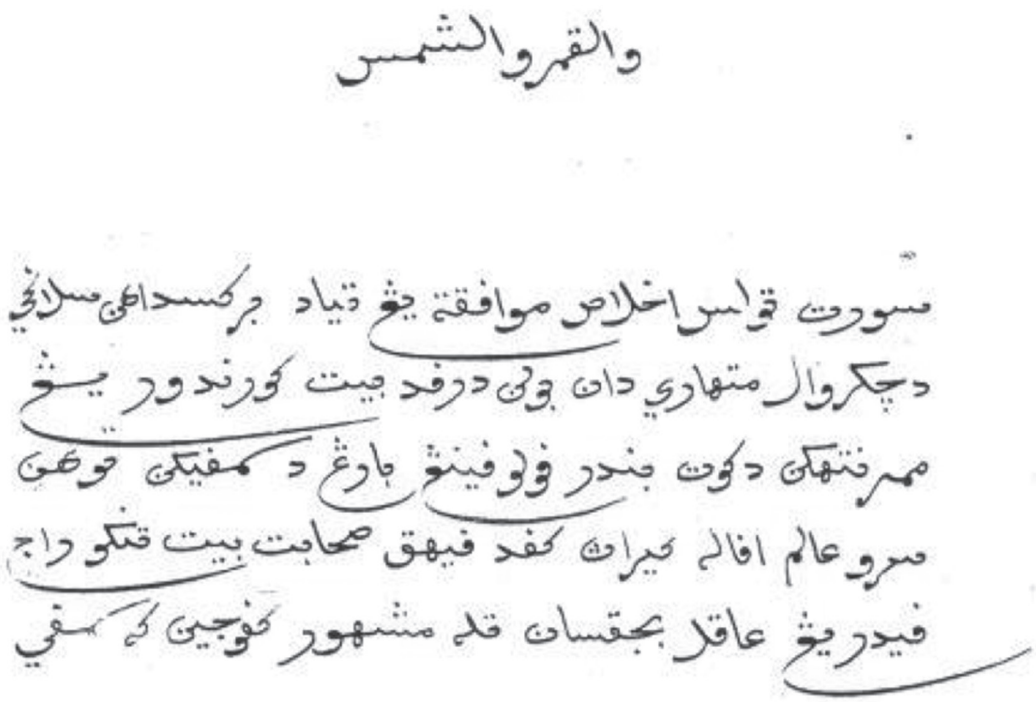

\section{REFERENCES}

Cabaton, Antoine, 1912. Catalogue sommaire des manuscrits indiens, indochinois et malayopolynésiens de la Bibliothèque nationale, Paris, Leroux.

Cardon, R.P.R., 1935. Le Roman de Pelandok le chevrotain, Bruges, Librairie de L'œuvre Saint-Charles.

Chambert-Loir, Henri, 1980a. « Catalogue des catalogues de manuscrits malais », Archipel 20, p. 45-69.

Chambert-Loir, Henri, 1980b. «Les manuscrits malais de Bâle, Lund, Singapour et Paris », Archipel 20, p. 87-98.

Chambert-Loir, Henri, et Fathurahman, Oman, 1999. Khazanah Naskah. Panduan Koleksi Naskah-Naskah Indonesia Sedunia. World Guide to Indonesian Manuscript Collections, Jakarta, EFEO-Yayasan Obor Indonesia.

Chambert-Loir, Henri, 1992. Compte-rendu de : « Katalog manuskrip Melayu di Perancis. Catalogue of Malay Manuscripts in France (Disusun oleh Siti Mariani Omar), Kuala Lumpur, Perpustakaan Negara Malaysia, 1991, viii + 208 p. », Bulletin de l'École française d'Extrême-Orient, Tome 79-1, p. 314-315.

Dulaurier, Édouard, [n.d.]. [Catalogue manuscrit inédit] (cité par Chambert-Loir et Fathurahman, 1999, p. 263).

Dulaurier, Édouard, 1843. Mémoire, lettres et rapports relatifs au cours de langues malaye et javanaise fait à la Bibliothèque royale pendant les années 1840-41, 1841-42, et à deux voyages littéraires entrepris en Angleterre, pendant les années 1838 et 1840, Paris, B. Duprat. 
Dulaurier, Édouard, éd., 1845. Lettres et Pièces Diplomatiques écrites en Malay... ( $1^{\mathrm{er}}$ fascicule), Paris, Firmin Didot Frères (cité par Chambert-Loir et Fathurahman, 1999, p. 264).

Dulaurier, Édouard, 1894 [Catalogue manuscrit, révision de Dulaurier [n.d.] par Henri-Léon Feer, inédit] (cité par Chambert-Loir et Fathurahman, 1999, p. 264).

Favre, Pierre, 1875. Dictionnaire malais-français, Vienne, Imprimerie impériale et royale.

Favre, Pierre, 1880. Dictionnaire francais-malais, Vienne, Imprimerie impériale et royale.

Favre, Pierre, 1883. « L'incendie de Singapour en 1828, poème malais de Abdullah ben Abdel-Kader », in Mélanges Orientaux, Paris, Ernest Leroux, p. 127-166.

Feer, Henri-Léon, 1898. « Introduction du nouveau catalogue des manuscrits malais-javanais de la Bibliothèque Nationale ", Revue des Bibliothèques 8, p. 371-380 [Introduction à Dulaurier 1894] (cité par Chambert-Loir et Fathurahman, 1999, p. 265).

Guillot, Claude, 1997. « À propos de François Albrand (1804-1867) et de son dictionnaire malais. Les Missions-Étrangères de Paris et la langue malaise au début du XIX ${ }^{\mathrm{e}}$ siècle », Archipel 54 (Destins croisés entre l'Insulinde et la France), p. 153-172.

Harun Mat Piah, 1982. «Dokumentasi : Manuskrip-manuskrip Cerita-cerita Panji Melayu di Bibliothèque Nationale de Paris » (Documentation : les manuscrits des histoires de Panji malaises de la Bibliothèque nationale de Paris), Jurnal Budaya Melayu, Volume 4, $\mathrm{n}^{\circ} 1$, p. $79-87$ (cité par Chambert-Loir et Fathurahman, 1999, p. 270).

Histoire des rois de Pasey, 2004 ( $2^{\mathrm{e}}$ éd.). Aristide Marre (traduit par) ; Monique Zaini-Lajoubert (présenté par), Toulouse, Anacharsis.

Katalog Manuskrip Melayu di Perancis. Catalogue of Malay Manuscripts in France. 1991. Siti Mariani Omar (établi par), Kuala Lumpur, Perpustakaan Negara Malaysia (Siri Bibliografi $\left.n^{\circ} 9\right)$.

Katalog Pameran Manuskrip Melayu Antarabangsa, Manuskrip Melayu : Suatu Penghayatan Minda Melayu 2-31 Oktober 1995 (Catalogue de l'exposition internationale de manuscrits. Les manuscrits malais : une appréciation de l'intellectualisme malais, 2-31 Octobre 1995), 1995. Kuala Lumpur, PNM (cité par Chambert-Loir et Fathurahman, 1999, p. 279).

Kratz, E.U., 1987. « Some Malay Letters on Trade », Indonesia Circle (44), p. 3-16.

Labrousse, Pierre, 1976. «Histoire des dictionnaires du malais et de l'indonésien », Archipel 12, p. $9-45$.

Labrousse, Pierre, 1995. " DULAURIER, Édouard », " FAVRE, Pierre, Étienne, Lazare », " MARRE DE MARIN, Aristide ", " TUGAULT, Alfred », "CABATON, Antoine ", in Pierre Labrousse, éd., Langues 'O 1795-1995. Deux siècles d'histoire de l'École des langues orientales, ouvrage publié avec le concours de la Caisse des dépôts et consignations, Paris, Éditions Hervas, p. 232-234, 234-235, 235-236, 236-237, 237-239.

Launay, A., 1916. Mémorial de la Société des Missions étrangères, Paris, Séminaire des Missions étrangères.

Le Calloc'h, B., 1995. «FEER, Léon », in Pierre Labrousse, éd., Langues 'O 1795-1995. Deux siècles d'histoire de l'École des langues orientales, ouvrage publié avec le concours de la Caisse des dépôts et consignations, Paris, Éditions Hervas, p. 270-271.

Lombard, Denys, 1976. « En mémoire de l'Abbé P. Favre », Archipel 12, p. 3-8.

Lombard, Denys, 1983. «Un grand précurseur : Antoine Cabaton (1863-1942) », Archipel 28, p. 17-24.

Makota Radja-Radja ou la couronne des rois par Bokhari de Djohore, traduit du malais et annoté par Aristide Marre, 1878. Paris, Maisonneuve.

Marre, Aristide, 1879. « Notice des manuscrits malais de la Bibliothèque Nationale de Paris ", Mémoires de la Société Académique Indo-Chinoise de France, I, Années 1877-1878, p. 174-188 (cité par Chambert-Loir et Fathurahman, 1999, p. 286).

Martin, Jean, 1886. Catalogue de la bibliothèque de la ville de Tournus (inédit).

Missions Étrangères et langues orientales : Contribution de la Société des Missions Étrangères 
à la connaissance de 60 Langues d'Asie : Bibliographie de 1680 jusqu'à 1996, 1997. Paris l'Harmattan et Missions Étrangères de Paris, (cité par Chambert-Loir et Fathurahman, 1999, p. 288).

Muhammad Haji Salleh, 1991. « Dua Orang Pengarang, Tiga Suara Gelap, dan Suatu SubGenre (Deux écrivains, trois voix clandestines et un sous-genre) », Archipel 42, p. 155176.

Muhammad Haji Salleh, éd., 1994. Syair Tantangan Singapura Abad Kesembilan Belas (Poèmes d'opposition de Singapour du dix-neuvième siècle), Kuala Lumpur, Dewan Bahasa dan Pustaka.

Pearson, J.D., 1971. Oriental MSS in Europe and North America; A Survey, Zug, Inter Documentation Company (Bibliotheca Asiatica 7) (cité par Chambert-Loir et Fathurahman, 1999, p. 293).

Proudfoot, Ian, 2001. «A "Chinese” Mousedeer Goes to Paris », Archipel 61, p. 69-97.

Voorhoeve, P., 1973. «Les manuscrits malais de la Bibliothèque nationale de Paris », Archipel 6, p. $42-80$.

Wilkinson, R.J., 1959. A Malay-English Dictionary (romanised), Londres, Macmillan \& Co Ltd, New York, St Martin's Press.

Zaini-Lajoubert, Monique, 2008. «Hikayat Raja Pasai dalam Bahasa Perancis Karya Terjemahan Aristide Marre (1874) » (Hikayat Raja Pasai en français, une traduction d'Aristide Marre (1874)), in Abdullah Hassan, Hasuria Che Omar, Dahlina Daut Mohmud, Sa'odah Abdullah, éds., Persidangan Penterjemahan Antarabangsa Ke-11, The $11^{\text {th }}$ International Conference on Translation, Membina Kepustakaan dalam Bahasa Melayu. Enriching the Repository of Knowledge in Malay, Kuala Lumpur, Institut Terjemahan Negara Malaysia Berhad (Institut national malaisien de la Traduction), p. 182-189. 
